\title{
ANÁLISIS DEL USO DE ESTRATEGIAS DE AFRONTAMIENTO EN LA ENFERMEDAD PULMONAR OBSTRUCTIVA CRÓNICA (EPOC): IMPLICACIONES CONCEPTUALES
}

\author{
ANTONIO GALÁN, M. ÁNGELES PÉREZ SAN GREGORIO y ALFONSO BLANCO \\ Departamento de Psiquiatría, Personalidad, Evaluación y Tratamiento Psicológicos, \\ Universidad de Sevilla
}

(Aceptado el 16 de octubre de 2000)

\begin{abstract}
El concepto de afrontamiento ocupa un lugar de primer orden en la investigación actual en el ámbito de la salud-enfermedad, pero se enfrenta a una situación de confusión conceptual que limita su operatividad. Hemos evaluado el uso de estrategias de afrontamiento y el estado afectivo en pacientes ambulatorios y hospitalizados que padecen una Enfermedad Pulmonar Obstructiva Crónica (EPOC) con un triple objetivo: a) obtener información sobre el uso de estrategias de afrontamiento y acerca del estado afectivo en los enfermos con EPOC; b) determinar los niveles de sensibilidad del concepto de Afrontamiento a los estresores que configuran las situaciones médicas; y c) establecer la capacidad de este concepto para predecir la presencia de alteraciones afectivas. Nuestros resultados nos han mostrado la debilidad del concepto de Afrontamiento, dada su falta de sensibilidad a los elementos que configuran la situación estresante y su incapacidad para recoger los factores moduladores del estado afectivo; hemos apuntado algunas explicaciones a esta situación y propuesto modificaciones en la definición y evaluación del Afrontamiento.
\end{abstract}

Palabras clave: Afrontamiento, Enfermedad Pulmonar Obstructiva Crónica (EPOC), alteraciones afectivas.

\section{Analysis of the use of coping at Chronic Obstructive Pulmonary Disease (COPD): conceptual implications}

Coping is at the first line at current research about health-illness, but it is facing a conceptual confusion that limits its efficiency. We have assessed the use of coping and the affective state in ambulatory and hospitalized Chronic obstructive pulmonary disease (COPD) patients, with three aims: a) getting of information about the use of coping and the affective states in COPD patients; b) determination of coping sensitivity levels to the stressors that configure medical situations; and c) establishment of capacity of this concept to predict the presence of emotional disorders. Our results have shown us the weakness of coping concept, now that its lack of sensitivity to the shaping items in the stressful situation and its incapacity to collect the modulating factors of the affective state; we have pointed out several explanations to this situation and proposed changes in coping evaluation and definition.

Key words: Coping, Chronic Obstructive Pulmonary Disease (COPD), emotional disorders.

\section{INTRODUCCIÓN}

\section{El concepto de Estrategia de Afronta- miento se ha revelado como uno de los principales instrumentos de abordaje de}

Correspondencia: Antonio Galán, Departamento de Psiquiatría, Personalidad, Evaluación y Tratamiento Psicológicos, Facultad de Psicología, Avenida San Francisco Javier s/n, 41005 Sevilla, Tfno.: 639884925 , Fax: 954557807. la realidad psicosocial del enfermar humano. Este concepto nació dentro de la teoría del estrés desarrollada por Lazarus y Folkman (1986); estos autores definieron el afrontamiento como «aquellos esfuerzos cognitivos y conductuales constantemente cambiantes que se desarrollan para manejar las demandas específicas externas $y / o$ internas que son evaluadas como excedentes o desbor- 
dantes de los recursos del individuo» (Lazarus y Folkman, 1986, p. 164). En el ámbito de la Psicología de la Salud ha sido utilizado como marco teórico sobre el que sustentar estudios que pretenden conocer el impacto de la enfermedad. Además, se ha usado como un objetivo de intervención psicológica, basándose en la supuesta capacidad del afrontamiento para modular los estados afectivos, influir en la cumplimentación de las prescripciones médicas, mejorar la calidad de vida, etc. (Ketelaars et al., 1996; Lazarus, 1993; López-Roig, Pastor y Rodríguez-Marín, 1993; Rodríguez-Marín, Pastor y López-Roig, 1993).

No obstante, nos encontramos ante un ámbito de estudio que se encuentra en un momento de confusión conceptual, especialmente por la falta de consenso en torno a qué actividades incluir bajo el rótulo de "Estrategia de afrontamiento»; en efecto, bajo éste se recogen desde patrones de actividad neuroendocrina hasta tipos específicos de procesamiento cognitivo. De esta forma, parece que nos situamos ante un megaconstructo en el que son incluidos fenómenos muy diferentes (Crespo y Cruzado, 1997; LópezRoig, 1991). Por otro lado, también existen dudas en torno a la idoneidad de los procedimientos de evaluación utilizados (Moix, 1990; Pelechano, 1992; Pelechano, Matud y de Miguel, 1993).

Uno de los ámbitos del enfermar menos abordados desde un punto de vista psicosocial es el de la patología respiratoria crónica, un área de trabajo en el que solamente el asma ha recibido atención. Frente a este cuadro, la Enfermedad Pulmonar Obstructiva Crónica (EPOC) ha recibido una escasa atención, a pesar del gran deterioro biopsicosocial que pueden llegar a experimentar los enfermos que la padecen.

Bajo la categoría nosológica de EPOC se agrupan un conjunto de enfermedades caracterizadas por la obstrucción cróni- ca al flujo aéreo (American Thoracic Society, 1987) y entre las que destacan la bronquitis crónica y el enfisema pulmonar. Los datos epidemiológicos muestran la gran importancia de estos trastornos. Así, se estima que la EPOC constituye la quinta causa de muerte más común entre los varones y la séptima para las mujeres (en total se le atribuyen unas 60 muertes al año por 100.000 habitantes de sexo masculino); además, en los últimos diez años se ha producido un incremento importante en estas tasas de mortalidad, de hasta un $50 \%$ entre los hombres (Comité Científico del Estudio IBERPOC, 1997). Centrándonos en las cifras de morbilidad, se estima que la EPOC genera por cada millón de habitantes cerca de 40.000 visitas ambulatorias, más de 3.000 ingresos hospitalarios, y aproximadamente 1.000 personas necesitan oxigenoterapia domiciliaria (Izquierdo, 1992).

En las fases iniciales de la EPOC, la sintomatología puede ser poco relevante o llamativa, con períodos ocasionales de tos o disnea ante esfuerzos de cierta consideración. Progresivamente, el paciente suele sufrir episodios de disnea ante esfuerzos cada vez menores, la tos se hace más frecuente y las expectoraciones resultan más abundantes, y es entonces cuando se acude al profesional sanitario. La evolución del cuadro es crónica, y suele conllevar un progresivo deterioro. Además, son múltiples las complicaciones que puede sufrir esta evolución, destacando especialmente la insuficiencia respiratoria aguda. El factor desencadenante más frecuente de estas descompensaciones son las infecciones respiratorias (Dorca y Estopà, 1985; Hafner y Ferro, 1999); así, se ha llegado a estimar que los pacientes con EPOC pueden presentar entre una y cuatro reagudizaciones por año, la mitad de las cuales se consideran de origen infeccioso. Esta doble necesidad, de realizar aportaciones a los 
problemas de definición en el ámbito del Afrontamiento, y de caracterizar a nivel psicosocial a los enfermos con EPOC, ha orientado esta investigación en la que pretendíamos cumplir con tres objetivos: (a) profundizar en el conocimiento de los factores psicosociales que caracterizan a este grupo de enfermos, evaluando las acciones que lleva a cabo cada individuo para enfrentarse a las situaciones estresantes relacionadas con su condición médica, y las manifestaciones ansiosas y depresivas que puede presentar; (b) determinar los niveles de sensibilidad del concepto de Afrontamiento a los estresores que configuran las situaciones médicas; y (c) conocer la relación entre el uso de determinadas estrategias de afrontamiento y aquellos factores que indican una buena o mala adaptación a la situación de enfermedad.

\section{MÉTODO}

\section{Participantes y procedimiento}

La muestra está compuesta por dos grupos de enfermos con EPOC. El primero de ellos estaba formado por 32 sujetos que se encontraban en un momento de estabilidad clínica y que acudían a una cita programada en una Unidad de Neumología. El segundo grupo, de 32 sujetos, lo componían enfermos que habían sufrido una descompensación de origen infeccioso y que se encontraban hospitalizados.

Como características generales para ambos grupos se requería ser varón, tener una edad comprendida entre 45 y 85 años, y no presentar alteraciones en sus aptitudes sensoriales o estado mental que les impidiesen orientarse espacio-temporalmente o mantener una conversación congruente. En todos los casos la selección de los enfermos se realizó en base al orden en que acudían o eran ingresados en las unidades en las que se llevó a cabo la investigación. Además, a todos los sujetos se les solicitó su colaboración voluntaria para responder a las entrevistas y cuestionarios, explicándoles que realizábamos un estudio acerca de los problemas de adaptación a la enfermedad y a los procedimientos médicos en situaciones como las suyas.

Los sujetos del primer grupo eran entrevistados por los investigadores en un despacho adyacente al utilizado por el especialista. La evaluación del segundo grupo se realizaba en la misma unidad de hospitalización, y se llevaba a cabo como mínimo 48 horas después del ingreso.

Fueron recogidas diversas variables sociodemográficas en las que, como nos indicaron los análisis posteriores, no existían diferencias intergrupales estadísticamente significativas. $Y$ así, para ambos grupos la edad media de los sujetos era de 64,37 años (Desviación típica de 7,55); se daba un predominio de sujetos que sólo sabían leer y escribir $(60,9 \%)$, que no trabajaban $(93,8 \%)$ y que estaban casados $(78,1 \%)$.

\section{Instrumentos}

La evaluación comenzaba con una Encuesta Sociobiográfica en la que recogimos algunas variables de carácter sociodemográfico y clínico. Tras ésta, se les administraban dos cuestionarios que pasamos a describir.

El Cuestionario de Estrategias de afrontamiento (CEA) fue elaborado por Rodríguez-Marín, Terol, López-Roig y Pastor (1992) a partir de trabajos previos que arrancan de lạ elaboración del Ways of Coping Questionnaire (WCQ) de Lazarus y Folkman (Folkman y Lazarus, 1985; Lazarus, 1990). Este cuestionario consta de 36 items con un formato de respuesta de entre 1 («Nunca») y 5 
( «Casi siempre, la mayoría de las veces"), en los que se recogen diversas conductas de afrontamiento ante una situación estresante.

La descomposición factorial del cuestionario dio lugar a la creación de once escalas: Pensamientos Positivos (interpretar el problema de manera ventajosa), Culpación de Otros (responsabilizar a otras personas del problema y/o de sus consecuencias), Pensamiento Desiderativo (expresar deseos de que no ocurra el problema y/o sus consecuencias), Búsqueda de Apoyo Social (solicitar ayuda), Búsqueda de Soluciones (obtención de información sobre la enfermedad, planificar y plantear posibilidades de solución), Represión Emocional (rechazo o evitación de la expresión de sentimientos o pensamientos), Contabilización de Ventajas (comparación de la situación actual con una posibilidad aun peor), Religiosidad (realización de prácticas religiosas), Autoculpación (responsabilizarse a sí mismo de la enfermedad), Resignación (aceptación de la situación como algo inamovible) y Escape (huida ante el problema).

Los autores indican que los resultados en cada dimensión deben obtenerse a partir de la media ponderada de los items que componen la escala, ajustándola a un sistema de puntuación de 0 a 100 que permita la comparación entre ellas.

El Hospital Anxiety and Depression Scale (HAD) fue desarrollado por Zigmond y Snaith (1983) intentando superar las dificultades que las medidas tradicionales de alteraciones emocionales se encontraban en el ámbito de la Psiquiatría de Enlace. Una de las principales dificultades de estas pruebas residía en la existencia de items que podían reflejar tanto síntomas de una enfermedad como manifestaciones ansiosas o depresivas (dolores de cabeza, pérdida de peso, etc.). Este instrumento ha recibido un extendi- do uso, mostrando unos índices de validez y fiabilidad muy satisfactorios (una excelente revisión de estos estudios se encuentra en Herrmann, 1997,y también en Moorey et al, 1991).

Este cuestionario consta de 14 items, siete referidos a la depresión y siete a la ansiedad, en los que se hace referencia a la forma en que el sujeto se ha sentido durante la última semana, debiendo elegir una de entre cuatro posibilidades de respuesta. La prueba proporciona dos valores, uno para la ansiedad y otro para la depresión, obtenidos a partir de la suma de las puntuaciones en cada subescala. Finalmente, el criterio de clasificación de las puntuaciones es el siguiente (Caro e Ibáñez, 1992): a) ausente de síntomas (0-7 puntos); b) caso dudoso (8-10 puntos); y c) presenta síntomas de depresion y/o ansiedad (11-21 puntos).

\section{RESULTADOS}

\section{Resultados relativos al uso de las estrategias de afrontamiento}

El primer paso de nuestro análisis consistió en el cálculo de las puntuaciones de los grupos en cada una de las once escalas. Una vez obtenidas, llevamos a cabo un contraste con el estadístico "t" para determinar en qué escalas existían diferencias intergrupales estadísticamente significativas. El análisis de estos datos, recogidos en la Tabla 1, nos muestra que sólo se presentan estas diferencias en la escala Pensamiento Desiderativo $(p=0,010)$, en donde destaca el mayor uso que de ella hacen los enfermos con EPOC hospitalizados ( $M=78,12)$ respecto a los ambulatorios $(M=56,44)$. En el resto de las escalas, las puntuaciones totales oscilan entre 9,08 (Culpación de Otros) y 79,49 (Contabilización de Ventajas). 
Tabla 1. Diferencias intergrupales en las dimensiones de afrontamiento

\begin{tabular}{lcccc}
\hline & \multicolumn{3}{c}{ Puntuaciones medias } & \\
\cline { 2 - 4 } \multicolumn{1}{c}{ Escala } & Ambulatorios & Hospitalizados & Totales & Significación estadística \\
\hline Pensamientos Positivos & 44,37 & $\mathbf{4 4 , 3 7}$ & $\mathbf{4 4 , 3 7}$ & $\mathbf{1 , 0 0 0}$ \\
Culpación de Otros & $\mathbf{1 2 , 1 1}$ & $\mathbf{6 , 0 5}$ & $\mathbf{9 , 0 8}$ & $\mathbf{0 , 1 6 0}$ \\
Pensamiento Desiderativo & $\mathbf{5 6 , 4 4}$ & $\mathbf{7 8 , 1 2}$ & $\mathbf{6 7 , 2 8}$ & $\mathbf{0 , 0 1 0 ^ { \star }}$ \\
Búsqueda de Apoyo Social & $\mathbf{2 1 , 8 7}$ & 27,08 & 24,48 & $\mathbf{0 , 4 7 5}$ \\
Búsqueda de Soluciones & $\mathbf{4 3 , 7 5}$ & 33,75 & 38,75 & $\mathbf{0 , 1 4 3}$ \\
Represión Emocional & $\mathbf{5 4 , 4 9}$ & $\mathbf{5 5 , 4 7}$ & $\mathbf{5 4 , 9 8}$ & $\mathbf{0 , 7 9 3}$ \\
Contabilización de Ventajas & $\mathbf{8 1 , 6 4}$ & $\mathbf{7 7 , 3 4}$ & $\mathbf{7 9 , 4 9}$ & $\mathbf{0 , 4 6 9}$ \\
Religiosidad & $\mathbf{3 0 , 8 6}$ & 30,08 & 30,47 & $\mathbf{0 , 9 0 6}$ \\
Autoculpación & $\mathbf{5 3 , 1 2}$ & $\mathbf{5 7 , 4 2}$ & $\mathbf{5 5 , 2 7}$ & $\mathbf{0 , 6 7 9}$ \\
Resignación & $\mathbf{6 7 , 9 7}$ & $\mathbf{8 0 , 8 6}$ & $\mathbf{7 4 , 4 1}$ & $\mathbf{0 , 0 8 1}$ \\
Escape & 20,31 & 16,14 & $\mathbf{1 8 , 2 3}$ & $\mathbf{0 , 3 5 7}$ \\
\hline
\end{tabular}

Nota: ${ }^{\star} \mathrm{p}<0,05$

Tabla 2. Diferencias intergrupales en las puntuaciones totales de ansiedad y depresión

\begin{tabular}{lcccc}
\hline & \multicolumn{3}{c}{ Puntuaciones medias } & \\
\cline { 2 - 4 } \multicolumn{1}{c}{ Escala } & Ambulatorios & Hospitalizados & Totales & Significación estadística \\
\hline Ansiedad & 5,719 & 7,187 & 6,453 & 0,297 \\
Depresión & 4,406 & 7,187 & 5,797 & $0,016^{\star}$ \\
\hline
\end{tabular}

Nota: ${ }^{*} \mathrm{p}<0,05$

Tabla 3. Clasificación en Ansiedad y Depresión según los parámetros de Caro e Ibáñez (1992)

\begin{tabular}{|c|c|c|c|c|c|c|}
\hline & \multicolumn{3}{|c|}{ Ansiedad } & \multicolumn{3}{|c|}{ Depresión } \\
\hline & Ausente & Dudosa & Presente & Ausente & Dudosa & Presente \\
\hline $\begin{array}{l}\text { Ambulatorios } \\
\text { Hospitalizados }\end{array}$ & $\begin{array}{l}22(68,8 \%) \\
19(59,4 \%)\end{array}$ & $\begin{array}{c}5(15,6 \%) \\
3(9,4 \%)\end{array}$ & $\begin{array}{c}5(15,6 \%) \\
10(31,3 \%)\end{array}$ & $\begin{array}{c}26(81,3 \%) \\
16(50 \%)\end{array}$ & $\begin{array}{l}4(12,5 \%) \\
6(18,8 \%)\end{array}$ & $\begin{array}{c}2(6,3 \%) \\
10(31,3 \%)\end{array}$ \\
\hline
\end{tabular}

Resultados en torno a la existencia de alteraciones afectivas

El análisis de las puntuaciones totales en las escalas de Ansiedad y Depresión nos proporcionó los resultados expuestos en la Tabla 2; tal como queda reflejado en ella, encontramos en ambas escalas que los enfermos hospitalizados obtienen puntuaciones más altas que los ambulatorios, si bien esta diferencia sólo resulta significativa en el caso de la Depresión $(p<0,05)$.

La clasificación de las manifestaciones afectivas en las categorías de "Ausente», «Dudosa» y "Presente» (Caro e Ibáñez, 1992) nos permite profundizar en el sig- nificado de las puntuaciones obtenidas en las dos dimensiones del cuestionario. Así, tal como queda reflejado en la Tabla 3 , se considera que aparecen claramente manifestaciones ansiosas en un 15,6\% de los enfermos ambulatorios y en un $31,3 \%$ de los hospitalizados. En el caso de la alteraciones depresivas las puntuaciones son de $6,3 \%$ y $31,3 \%$ respectivamente.

El análisis de las diferencias intergrupales en esta clasificación fue realizado mediante la prueba de Mann-Whitney (Tabla 4), con unos resultados similares a los obtenidos en el análisis de las puntuaciones totales de las escalas: aparecen más 
Tabla 4. Prueba de Mann-Whitney ( $U$ ) con los datos de clasificación de los sujetos en ansiedad y depresión

\begin{tabular}{ccccc}
\hline Variable & $\begin{array}{c}\text { Ambulatorios } \\
\text { (Rango medio) }\end{array}$ & $\begin{array}{c}\text { Hospitalizados } \\
\text { (Rango medio) }\end{array}$ & $U$ & $\begin{array}{c}\text { Significación } \\
\text { estadística }\end{array}$ \\
\hline Ansiedad & 30,45 & 34,55 & 446,5 & 0,301 \\
Depresión & 27,06 & 37,94 & 338,0 & $0,005^{* *}$ \\
\hline
\end{tabular}

Nota: ${ }^{\star \star} \mathrm{p}<0,01$.

Tabla 5. Parámetros del modelo de regresión

\begin{tabular}{lccccc}
\hline & R Múltiple & $R^{2}$ & $R^{2}$ ajustada & $\begin{array}{c}\text { Error } \\
\text { estandarizado }\end{array}$ & Signif. $F$ \\
\hline Ansiedad & 0,375 & 0,140 & 0,112 & 5,266 & $0,009^{\star *}$ \\
Depresión & 0,284 & 0,081 & 0,066 & 4,494 & $0,023^{*}$ \\
\hline
\end{tabular}

Nota: ${ }^{*} \mathrm{p}<0,05 ;{ }^{\star *} \mathrm{p}<0,01$.

alteraciones afectivas en los enfermos hospitalizados, si bien estas diferencias sólo resultan estadísticamente significativas en el caso de la Depresión ( $p<0,01$ ).

\section{Resultados del Análisis de Regresión Múltiple}

Llevamos a cabo un Análisis de Regresión utilizando como variables predictoras las escalas que configuran el CEA y como variables criterio las puntuaciones totales en las escalas de Ansiedad y Depresión, obteniendo así dos ecuaciones de regresión.

Antes de realizar este análisis, juzgamos necesario valorar la idoneidad de este procedimiento en función de nuestros datos. Para ello, y a través de un análisis gráfico de los residuales estandarizados, comprobamos que se cumplían las condiciones de linealidad y homocedasticidad de los datos. Por otro lado, la independencia de los errores fue evaluada a través del estadístico de Durbin-Watson (Ansiedad: 2,055; Depresión: 1,907). Finalmente, la normalidad de la distribución de los residuos fue comprobada a través de la prueba de Kolmogorov-Smir- nov con las puntuaciones residuales estandarizadas (Ansiedad: $p=0,132$; Depresión: $p=0,386$ ).

Tras comprobar el cumplimiento de estos requisitos, abordamos el Análisis de Regresión Múltiple con el procedimiento "Stepwise», lo que nos proporcionó ecuaciones de regresión estadísticamente significativas para las puntuaciones en Ansiedad ( $p<0,01$ ) y Depresión ( $p<0,05)$ (Tabla 5). Los valores $R$ nos muestran que la capacidad predictiva sobre las alteraciones afectivas es mayor en el caso de la Ansiedad $\left(R^{2}\right.$ ajustada $\left.=0,112\right)$ que en la Depresión ( $R^{2}$ ajustada $=0,066$ ), obteniéndose en ambos casos una puntuación muy baja.

Una vez establecida la capacidad de los datos para predecir la existencia de alteraciones afectivas, abordamos la identificación de las escalas del CEA que permiten realizar estas predicciones. En el caso de la Ansiedad (Tabla 6), sólo dos dimensiones lo permitían: Pensamiento Desiderativo $(p<0,05)$ y Culpación de otros $(p<0,05)$. En ambos casos, la asociación entre las variables es positiva (un mayor uso de las estrategias se relaciona con una mayor presencia de sintomato- 
logía ansiosa). Vemos por tanto que en ningún caso podemos identificar estrategias que se asocien a menores índices de alteraciones de carácter ansioso.

Respecto a la sintomatología depresiva (Tabla 6), el análisis de regresión sólo recoge una dimensión relacionada con ella: Pensamientos Positivos ( $\mathrm{p}<0,05$ ). Atendiendo al valor $T$, podemos considerar que el uso de esta estrategia de afrontamiento se asocia a una menor presencia de sintomatología depresiva.

\section{DISCUSIÓN}

El análisis de la información descriptiva en torno a las estrategias de afrontamiento nos permite comprobar el predominio de aquellas actividades que implican la aceptación del enfermar como una situación inevitable. En efecto, las estrategias más utilizadas implican la búsqueda de adaptación a un estado médico asumido. Así, los enfermos se esfuerzan en buscar aspectos positivos a su situación (intentar ver el lado bueno, considerar las ventajas, concentrarse en cosas buenas, etc.) y tratan de aceptar su enfermedad considerando que no es posible hacer nada. Frente a estas estrategias, se sitúan en un segundo plano aquellas que implican la búsqueda de soluciones al problema, la petición de apoyo social o el alejamiento de la situación estresante. Estamos por tanto ante esfuerzos de adaptación a una enfermedad aceptada como crónica y que demanda, más que una solución, una acomodación satisfactoria.

Otro resultado destacable ha sido la constatación de los importantes niveles de alteraciones afectivas que presentan los enfermos con EPOC. La clasificación de los sujetos en función de sus alteraciones ansiosas y depresivas nos da una indicación de la alta prevalencia de éstas, y nos permite apreciar cómo aumenta cuando se produce una descompensación del cuadro. Además, estos datos nos remiten a los existentes en la bibliografía en torno a las importantes manifestaciones ansiosas y depresivas que aparecen en los enfermos con EPOC (Anderson, 1995; Araúxo, Sureda y Pablo, 1997; Creer, 1983; Lisansky y Clough, 1996; Quirk y Jones, 1995; Van Elderen, 1995). Así, los trabajos previos no sólo nos informan de la alta prevalencia en estos enfermos sino también de las diferencias que existen entre los síntomas depresivos y ansiosos, con una mayor presencia de aquellos.

Centrándonos en el ámbito del Afrontamiento, y atendiendo más allá de las conclusiones que podamos establecer en torno a los grupos muestrales utilizados en esta investigación, nuestros resultados aportan información que puede resultar valiosa en los debates existentes en torno al estatus conceptual de este constructo. Como ya planteamos, estamos ante un concepto profusamente utilizado en el ámbito de la salud-enfermedad, pero ha recibido cuantiosas críticas y han sido

Tabla 6. Variables en las ecuaciones de regresión

\begin{tabular}{|c|c|c|c|c|c|c|}
\hline Escala & Variable & $B$ & $S E B$ & Beta & $T$ & Sig. $T$ \\
\hline \multirow{3}{*}{ Ansiedad } & Culpación de Otros & 0,0802 & 0,0386 & 0,2465 & 2,076 & $0,042^{*}$ \\
\hline & Pensam. Desiderativo & 0,0453 & 0,0195 & 0,2754 & 2,319 & $0,024^{\star}$ \\
\hline & (Constante) & 2,6743 & 1,5036 & & 1,779 & 0,080 \\
\hline \multirow[t]{2}{*}{ Depresión } & Pensam. Positivos & $-0,0528$ & 0,0226 & $-0,2842$ & $-2,334$ & $0,023^{*}$ \\
\hline & (Constante) & 8,1415 & 1,1508 & & 7,074 & $0,000 * * *$ \\
\hline
\end{tabular}

Nota: ${ }^{\star} \mathrm{p}<0,05 ;{ }^{* *}{ }^{*} \mathrm{p}<0,001$ 
generadas formulaciones discrepantes con su concepción tradicional. Nuestros análisis han arrojado dos bloques de resultados que contradicen la definición de Lazarus y Folkman (1986). Son los siguientes:

\section{A) Incapacidad para recoger los cambios en la situación de enfermar}

En la definición de afrontamiento se enfatiza el carácter situacional de las estrategias puestas en funcionamiento ante el estresor (Lazarus y Folkman, 1986), pero en nuestra investigación hemos recogido las estrategias utilizadas en dos situaciones médicas muy distintas (por el nivel de gravedad, por la hospitalización, etc.) y no hemos hallado patrones diferenciales de afrontamiento. Este resultado podría ser interpretado desde dos enfoques distintos:

1. La inadecuación de la conceptualización del afrontamiento: Así, en primer lugar podríamos plantear que esta concepción tradicional no recoge algunas conductas que podrían ser relevantes y que darían cuenta de las conductas que el sujeto pone en funcionamiento en las situaciones estresantes. En este sentido, autores como Moix (1990) y Moos (1982) demandan la necesidad de incluir en las estrategias de afrontamiento las conductas automatizadas. En efecto, es posible que ante la enfermedad, algunas personas pongan en funcionamiento conductas que, al ser utilizadas de manera habitual, no requieran la realización de un esfuerzo (y por tanto no serían conductas de afrontamiento propiamente dichas). Esto resultaría especialmente relevante en el caso de enfermedades crónicas, en las que las estrategias de afrontamiento relativas a la salud formarían parte de la rutina de funcionamiento del individuo. En una línea similar algunos autores plantean una concepción más rasguista y menos situacional del afrontamiento (Ferrero, 1993; Pelechano, 1992, 1997). Desde este punto de vista, las estrategias de afrontamiento no tendrían por qué cambiar de una situación estresante a otra, lo que justificaría los resultados de nuestra investigación; en efecto, nos encontraríamos ante dos grupos de enfermos muy similares que sólo se diferenciarían entre sí por la aparición puntual de una descompensación en uno de los grupos; en cuanto que las estrategias de afrontamiento serían rasgos relativamente estables, éstas permanecerían a través de este cambio.

2. La inadecuación de la evaluación del afrontamiento: Los instrumentos habituales de evaluación del afrontamiento han recibido numerosas críticas, y muchas de ellas pueden ser aplicables al instrumento utilizado por nosotros. Así, Moix (1990) plantea la especificidad de la población anciana, en la que instrumentos elaborados con jóvenes no resultan útiles. O la planteada por Pelechano (1992) y Pelechano et al. (1993) acerca de la sobreabundancia de estudios con estudiantes, circunstancia que limita su aplicabilidad a poblaciones diferentes a ésta. Finalmente, debemos cuestionarnos la conveniencia de usar el Análisis Factorial en la creación del instrumento. Así, Aliaga y Capafons (1996) consideran que este procedimiento estadístico resulta inadecuado conceptualmente para este tipo de datos. En efecto, el carácter situacional del afrontamiento hace que la aplicación a éste del Análisis Factorial genere artefactos debidos a los cambios de situación. De la misma manera, el Análisis Factorial enfatiza que un 
ítem debe pertenecer a un sólo factor o escala, a pesar de que una estrategia puede cumplir distintas funciones ante cada uno de los rasgos que componen una situación estresante (Moix, 1990).

\section{B) Incapacidad para predecir la pre- sencia de alteraciones afectivas en los enfermos}

Habitualmente las estrategias de afrontamiento han sido consideradas como variables predictoras de múltiples criterios, como el estado afectivo, la recuperación o la adherencia a los tratamientos (Ketelears et al., 1996; Lazarus, 1993; López-Roig et al., 1993; Rodríguez-Marín et al., 1993). De una forma especial ha destacado la importancia concedida a su potencial capacidad para atenuar el deletéreo efecto de algunos estresores (Heszen-Niejodek, 1995; Lazarus, 1993). Nuestro análisis se centró en las alteraciones afectivas, más concretamente en la aparición de síntomas ansiosos y depresivos; los resultados han sido negativos, en cuanto que apenas existen dimensiones de afrontamiento que predigan la aparición de alteraciones afectivas. En este sentido, nuestros datos apoyan las dudas planteadas por algunos autores en torno a la eficacia de las estrategias de afrontamiento (Carver y Scheier, 1994; Moix, 1990).

Tras analizar nuestros resultados y la bibliografía previa en torno a este tema, quisiéramos finalizar este análisis del concepto de Estrategia de Afrontamiento planteando algunas objeciones a la forma habitual de definirlo y evaluarlo, intentando así arrojar cierta luz sobre las debilidades que diversos autores y nuestro trabajo han formulado. Son las siguientes:

1. En primer lugar, podríamos llamar la atención sobre cierto carácter indiscriminado en la identificación de las estrategias, en el sentido de que frecuentemente son incluidas entre éstas algunas conductas sin un componente intencional o funcional respecto a la situación estresante. En efecto, tal como plantea la definición de Lazarus, las estrategias de afrontamiento son actividades "que se desarrollan para manejar» (Lazarus y Folkman, 1986, p. 164) una situación determinada. Pues bien, con frecuencia, bajo el rótulo de "estrategia» parecen recogerse resultados del proceso de afrontamiento más que éste en sí, o actividades que un sujeto puede realizar sin un componente funcional claro respecto a los estímulos que le rodean.

2. En segundo lugar, y de manera inversa, apreciamos una ausencia de cierto tipo de conductas, como las automáticas (Moix, 1990) o de autocontrol (Aliaga y Capafons, 1996). Son conductas que, a pesar de resultar excluidas de la definición proporcionada por el grupo de Lazarus, se ajustan intuitivamente al concepto que ésta intenta apresar.

3. En tercer lugar, debemos plantearnos hasta qué punto la falta de relación existente entre las estrategias de afrontamiento y otras variables de resultado, como el estado afectivo, tiene su origen en la falta de consenso en el ámbito teórico e investigador. En efecto, son variadísimos los instrumentos y las poblaciones médicas en las que se ha estudiado la eficacia del afrontamiento, de tal forma que resulta difícil generalizar los resultados y tener así un fundamento firme sobre el que valorar la eficacia global del concepto que nos ocupa. Por otro lado, algunos autores (por ejemplo Cohen y Lazarus, 1994) anotan la 
falta de finura conceptual en el ámbito del afrontamiento, de tal forma que bajo un mismo rótulo se incluyen procesos que podrían ser muy diferentes.

Teniendo en cuenta todos estos puntos, podemos concluir lo siguiente: (1) Los enfermos con EPOC sufren elevados niveles de ansiedad y depresión, especialmente ante descompensaciones del cuadro médico, y (2) el concepto de «Estrategia de Afrontamiento" muestra importantes limitaciones en su aplicación al mundo del enfermar; así, no permite recoger los cambios que se producen en la situación de enfermar o predecir la presencia de alteraciones afectivas, lo que atribuimos a una inadecuación de las estrategias habitualmente consideradas. En este sentido, consideramos necesario realizar un esfuerzo por delimitar aquellas actividades que de forma continuamente cambiante realizan los individuos para adaptarse a la evolución de su enfermedad.

\section{REFERENCIAS BIBLIOGRÁFICAS}

Aliaga, F., y Capafons, A. (1996). La medida del afrontamiento: revisión crítica de la "Escala de Modos de Coping" (Ways of Coping). Ansiedad y Estrés, 2, 17-26.

American Thoracic Society (1987). Standards for the diagnosis and care of patients with chronic obstructive pulmonary disease (COPD). American Review of Respiratory Disease, 136, 225-244.

Anderson, K.L. (1995). The effect of chronic obstructive pulmonary disease on quality of life. Research in Nursing and Health, 18, 547-556.

Araúxo, A., Sureda, B., y Pablo, J. de. (1997). Sistema respiratorio. En J.E. Rojo y E. Cirera (Eds.), Interconsulta psiquiátrica (pp. 219-231). Barcelona: Masson.

Caro, I., e Ibáñez, E. (1992). La Escala Hospitalaria de Ansiedad y Depresión. Su utilidad práctica en Psicología de la Salud. Boletín de Psicología, 36, 43-69.
Carver, C.S., y Scheier, M.F. (1994). Situational coping and coping dispositions in a stressful transaction. Journal of Personality and Social Psychology, 66, 184-195.

Cohen, F., y Lazarus, R.S. (1982). Coping with the stresses of illness. En G.C. Stone, F. Cohen y N.E. Adler (Eds.), Health Psychology. A handbook (pp. 217-254). San Francisco: Jossey-Bass.

Comité Científico del Estudio IBERPOC. (1997). Proyecto IBERPOC: un estudio epidemiológico de la EPOC en España. Archivos de Bronconeumología, 33, 293-299.

Creer, T.L. (1983). Respiratory disorders. En T.G. Burish y L.A. Bradley (Eds.), Coping with chronic disease. Research and applications (pp. 313-337). London: Academic Press.

Crespo, M., y Cruzado, J.A. (1997). La evaluación del afrontamiento: adaptación española del cuestionario COPE con una muestra de estudiantes universitarios. Análisis y Modificación de Conducta, 23, 797-830.

Dorca, J., y Estopà, R. (1985). Insuficiencia respiratoria. Factores desencadenantes. Clínica. Medicine (Ed. Española), 27, 3845.

Ferrero, J. (1993). El afrontamiento de un diagnóstico de cáncer. Valencia: Promolibro.

Folkman, S., y Lazarus, R.S. (1985). If it changes it must be a process: study of emotion and coping during three stages of a college examination. Journal of Personality and Social Psychology, 48, 150-170.

Hafner, J.P., y Ferro, T.J. (1999). Bronquitis aguda en adultos: enfoque terapéutico actual. Tiempos médicos, 556, 7-14.

Herrmann, C. (1997). International experiences with the Hospital Anxiety and Depression Scale. A review of validation data and clinical results. Journal of Psychosomatic Research, 42, 17-41.

Heszen-Niejodek, I. (1995). The level of patients ${ }^{\prime}$ anxiety and their illness behavior. En J. Rodríguez-Marín (Ed.), Health Psychology and Quality of Life research (Vol. 1, pp. 396-404). Alicante: Departamento de Psicología de la Salud.

Izquierdo, J. (1992). Enfermedad pulmonar obstructiva crónica. Conceptos, definicio- 
nes y substrato patológico. En J. Morera (Ed.), Enfermedad Pulmonar Obstructiva Crónica: Vol. 1. Conceptos generales (pp. 1-12). Barcelona: MCR.

Ketelaars, C.A., Schlosser, M.A., Moster, R., Huyer-Abu-Saad, H., Halfens, R.J., Wouters, E.F. (1996). Determinants of healthrelated quality of life in patients with chronic obstructive pulmonary disease. Thorax, 51, 39-43.

Lazarus, R.S. (1990). Stress, coping and illness. En H.S. Friedman (Ed.), Personality and disease (pp. 97-120). New York: Wiley.

Lazarus, R.S. (1993). From psychological stress to the emotions: a history of changing outlooks. Annual Review of Psychology, 44, 1-21.

Lazarus, R.S., y Folkman, S. (1986). Estrés y procesos cognitivos. Barcelona: Martínez Roca. (Original en inglés, 1984).

Lisansky, D.P., y Clough, D.H. (1996). A cognitive-behavioral self-help educational program for patients with COPD. Psychotherapy and Psychosomatics, 65, 97-101.

López-Roig, S. (1991). Determinantes psicosociales del estrés y su afrontamiento en pacientes quirúrgicos. Tesis Doctoral no publicada, Universidad de Alicante.

López-Roig, S., Pastor, M.A., y RodríguezMarín, J. (1993). Programas de preparación psicológica para la cirugía. En F.X. Méndez, D. Maciá y J. Olivares (Eds.), Intervención conductual en contextos comunitarios $I$. Programas aplicados de prevención (pp. 169-215). Madrid: Pirámide.

Moix, J. (1990). Estrategias de afrontamiento: consideraciones críticas sobre su concepto y sus formas de medición. Psicologemas, 4, 193-205.

Moorey, S., Greer, S., Watson, M., Gorman, C., Rowden, L., Tunmore, R., Robertson, B., y Bliss, J. (1991). The factor structure and factor stability of the Hospital Anxiety and Depression Scale in patients with cancer. British Journal of Psychiatry, 158, 255-259.
Moos, R.H. (1982). Coping with acute health crises. En T. Millon, C. Green y R. Meagher (Eds.), Handbook of Clinical Health Psychology (pp. 129-151). New York: Plenum Press.

Pelechano, V. (1992). Personalidad y estrategias de afrontamiento en pacientes crónicos. Análisis y Modificación de Conducta, 18, 167-202.

Pelechano, V. (1997). Personalidad y el binomio salud-enfermedad: una revisión conceptual selectiva. Análisis y Modificación de Conducta, 23, 751-795.

Pelechano, V., Matud, P., y de Miguel, A. (1993). Habilidades de afrontamiento en enfermos físicos crónicos. Análisis y Modificación de Conducta, 19, 91-146.

Quirk, F.H., y Jones, P.W. (1995). Preliminary validity of two, new, «short» airways questionnaires. En J. Rodríguez-Marín (Ed.), Health Psychology and Quality of Life research (Vol. 2, pp. 693-703). Alicante: Departamento de Psicología de la Salud.

Rodríguez-Marín, J., Pastor, M.A., y LópezRoig, S. (1993). Afrontamiento, apoyo social, calidad de vida y enfermedad. Psicothema, 5, 349-372.

Rodríguez-Marín, J., Terol, M.C., LópezRoig, S., y Pastor, M.A. (1992). Evaluación del afrontamiento del estrés: propiedades psicométricas del Cuestionario de Formas de Afrontamiento de Acontecimientos Estresantes. Revista de Psicología de la Salud, 4, 59-84.

Van Elderen, T. (1995). Quality of life in patients with cancer, CNSLD, coronary heart disease and diabetes mellitus: a review of research in the Netherlands. En J. Rodríguez-Marín (Ed.), Health Psychology and Quality of Life Research (Vol. 1, pp. 356-373). Alicante: Departamento de Psicología de la Salud.

Zigmond, A.S., y Snaith, R.P. (1983). The Hospital Anxiety and Depression Scale. Acta Psychiatrica Scandinavica, 67, 361370. 